\title{
Evaluation of the Risk Problem and the Selection of the Optimum Risk Management Solution
}

CRAIG VAN ANNE

The Hartford Steam Boiler Inspection and Insurance Company

\section{ABSTRACT}

The determination of optimum fire protection engineering solutions at present is a predominantly subjective based process. An analytical technique is offered to evaluate risk and the manager's aversion toward risk to better employ the risk management options of loss transfer, loss absorption, and loss reduction.

An engineering method presently exists for numerically evaluating a relative level of risk in any building. The flame movement part of the method involves the determination of the probability of success in terminating a fire within each space of a building. The effectiveness of each barrier surrounding the space, also can be evaluated whether it be a floor, ceiling, wall, or an empty void.

An illustrative case study has been offered to demonstrate the incorporation of the engineering method into a decision analysis of possible alternatives based on the risk attitude of the decision maker. This case study illustrates how the three risk management options can be considered rationally and quantitatively.

\section{THE ENGINEERING METHOD AND RISK MANAGEMENT}

There exists today a detailed engineering method (1) which, regardless of size or occupancy, can evaluate in a consistent manner any building. Through the application of this engineering method, a COPE (*) evaluation is performed which yields relative assessments of risk. Once the fire risk of a particular building is quantified, the appropriate parties can analyze possible risk management solutions. The evaluation of these components yields a probability value in the form of an L-curve. The L-curve is a graphical representaion of the cumulative probability (from 0.0 to 1.0 ) that a fire will be limited to the space being considered. Limited, in this sense, means that the fire will not propagate beyond the area which has been evaluated. Figure 1 shows typical L-curves.

This paper will concentrate its use of the engineering method on the Flame Movement Analysis. We wish to evaluate the likelihood that a fire will be limited to an area of a building. This likelihood is based on four engineering method parameters which evaluate COPE: an evaluation of the hazard present --the I curve; the automatic suppression system--the A-curve; manual fire

fighting--the M-curve, and barrier effectiveness; either physical or spatial.

(*) COPE is acronym for the class of construction, occupancy, installed fire protection systems, and external exposures of a building. 
An evaluation of a barrier is dependent on the specific type of fire that will attack the barrier, and of course barrier construction. A barrier can either be physical, or a space separation. A fire must penetrate the barrier to cause ignition in the next space.

\section{RISK MANAGEMENT APPLICATIONS}

By simply inserting a zero value for any L-curve component (I-, A-, $M-, \& B$ ) into a network calculation, the effect that that component has in the L-curve can be negated. The ease of this "what if" situation capability is ideal for an effective comparison of various loss prevention alternatives and the resources required to implement them.

The extent of loss under different risk management situations is of primary importance if risk decisions are to be made. Loss estimates of the various risk management parameters can be easily assessed with the aid of the final product of the engineering method: the $L-c$ urve. This paper will address NLE and MFL risk management parameters using the L-curve..

\section{NLE and MFL CURVES}

The risk management parameters commonly used are Normal Loss expectancy (NLE) and Maximum Foreseeable Loss (MFL). Though these terms are used widely by those professionals dealing with risk management, definitions of each parameter are not widely consistent. Therefore, definitions of the risk management parameters as used in this paper will be given.

\section{NLE Curve}

The NLE is an evaluation of the normal loss to be expected when automatic suppression systems and public or private fire fighting assistance is present and fully functional. An engineering method generated NLE curve would then simply be the unaltered L-curve. In this situation the A-curve, M-curve, I-curve, and barrier performance could a11 have values greater than zero.

\section{MFL Curve}

The absolute worst risk management parameter is described by an MFL estimate. This estimate considers all automatic fire protection systems are impaired, and no manual fire fighting assistance is received. Any fire spread limitation offered by interior fire separation walls is completely ignored unless a MFL $\left.{ }^{*}\right)$ fire wall is present. The engineering method MFL curve only considers the fire protection implications of loss due to the fire peril presented.

The engineering method generates an MFL-curve by assigning zero values to the A-curve, M-curve and barrier performance. Should an MFL wall be present, the values then assigned to Barrier Performance would be 1.0. Therfore, a network calculated MFL-curve can take only two shapes: that of the I-curve or that of the accumulated I-curve and barrier performance values. Figure 1 summarizes the risk management curves just discussed.

(*) MFL fire wall as defined by Loss Prevention Data Sheet 1-22, Factory Mutual Engineering Corporation. 
BASIC CURVES

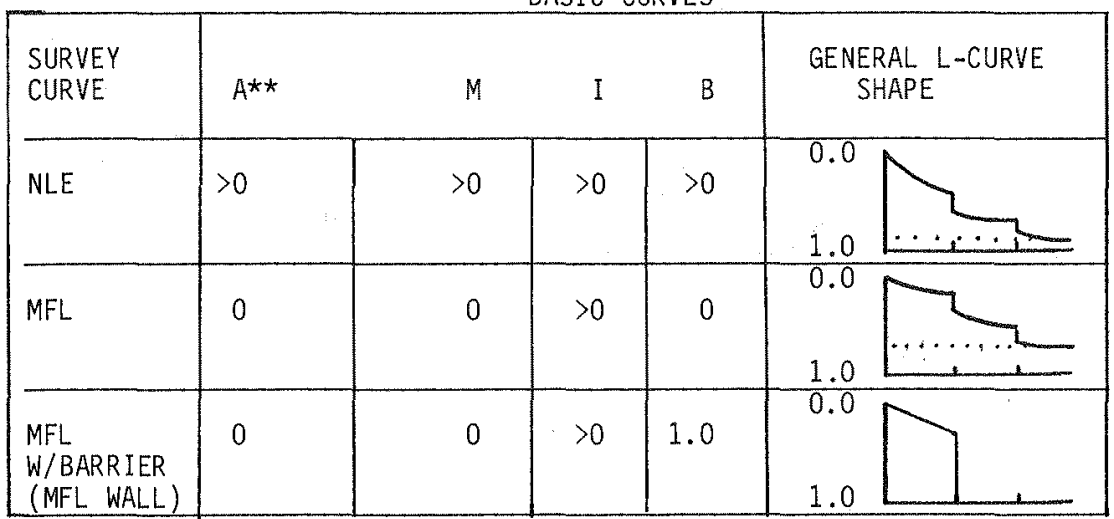

** BEYOND ROOM OF ORIGIN, AUTOMATIC SUPPRESSION ASSUMED

INEFFECTIVE: $A$-CURVE $=0.0$

\author{
Figure 1 NLE, MFL \\ RISK MANAGEMENT CURVES
}

\title{
DECISION ANALYSIS AND THE ATTRACTIVE ALTERNATIVE
}

Engineering method generated probabilities can be used together in a decision analysis model to determine what course of action to take for a simple "two room" building. In conjunction with this mode1, a major parameter in choosing an alternative is the decision maker's risk attitude.

The rooms in this example could be of any size that would meet the objectives of the analysis. Figure 2 is a simple plan of this example building. The objective of this illustration is to show the overall process of applying decision analys is using probabilities generated by the engineering method. Therefore, the details of the construction, occupancy and protection of this two room building are not imperative for illustrative purposes. The

\begin{tabular}{|c|c|}
\hline ROOM 1 & ROOM 2 \\
AREA $=10,000 \mathrm{FT} 2$ & AREA $=10,000 \mathrm{FT} 2$ \\
$\mathrm{I}=.4, A=.85, M=.1$ & $\mathrm{I}=.6, A=0, M=.4$ \\
\hline
\end{tabular}

Figure 2 Case Study Floor Plan 
same analytic process used in this simplistic approach would be used in the more complicated risks found in actual practice. Room 2 of Figure 2 has been identified as the area of concern based on the highest loss potential in dollars of value. A fire is assumed to start in Room 1 and proceed from left to right. If the fire penetrates the barrier, it must propagate to Room 2 .

This is a simple condition for the engineering method. In more complicated situations, the engineering method's computer program (2) is capable of identifying the worst case scenario fire which would pose the greatest threat to any room or area of interest in the building.

\section{FIELD SURVEY}

An evaluation of this building has provided "engineering method data" which yields the assessments of Table 1 . This table represents the worst case scenario based on data supplied by the evaluating engineer. In this example, Room 1 has been identified as the ROOM OF ORIGIN b ecause it has the lowest probability of a fire self-extinguishing: $P(I)=0.4$. The computer program developed for the engineering method can easily determine the worst ROOM OF ORIGIN, or be instructed to accept a particular room of origin for a "what-if" analysis.

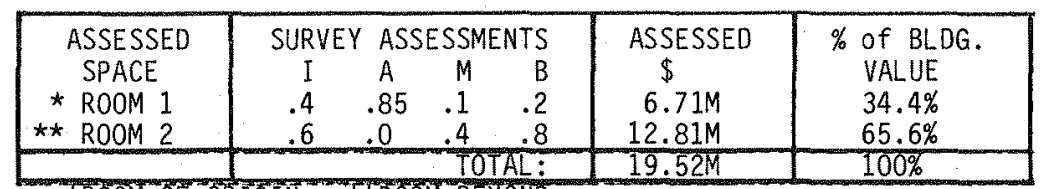

*ROOM OF ORIGIN

Table 1 SURVEY DATA

This table represents the worst case scenario based on data supplied by the evaluating engineer. In this example, Room 1 has been identified as the ROOM OF ORIGIN b ecause it has the lowest probability of a fire self-extinguishing: $P(I)=0.4$. The computer program developed for the engineering method can easily determine the worst ROOM OF ORIGIN, or be instructed to accept a particular room of origin for a "what-if" analysis.

Room 2 is a ROOM BEYOND THE ROOM OF ORIGIN (ROOM BEYOND) and is also an object of concern for this evaluation as it reprsents the greatest loss potential. Room 2 represents $66 \%$ of the exposed value of this building; a nything from a warehousing area to a computer room. As above, a specific ROOM BEYOND : an be assigned in the computer program.

The manner in which the survey assessments of Table 1 were determined is not important to the present objective. This assessment would have used a normal application of the engineering method. Therefore, in order to provide an illustration of the decision analysis process, the actual details of construction, occupancy, protection and exposure used in decomposed probability assessments (3) can be assumed to have been performed by the engineering analysis in order to provide an illustration of the decision analysis process.

The probability of limiting a fire at individual rooms is obtained from network diagrams of the engineering method. With this probability we can 
then determine the probability of limiting the fire to " $Z$ " sq.ft. in space $X$, given the fire was not limited to " $Y$ " $s q$. $f t$. in space $X$, where $Z$ is greater than $Y$ :

$$
\begin{aligned}
& P(L 1 @ Z / L 1 @ Y) \text { Where } L=\text { not limited } \\
& \text { For example, }
\end{aligned}
$$

$$
\begin{aligned}
& P(L 1 \cap 3,000 / \text { NLE FIRE })=.919 \\
& P(L 1 \odot 6,000 / L 1 \odot 3000)=8.1 \times 10-6=0
\end{aligned}
$$

are obtained from an evaluation of Room 1 using the engineering method for the segmented floor areas. Likewise, this same evaluation would yield sector probabilites for Room 2. Sector areas are shown in Figure 3.

The seemingly unwarranted number of significant figures are included in Figure 3 to illustrate a conservative assumption. If a fire is limited to

\begin{tabular}{|c|c|}
\hline EVENT SECTOR & $P(L)$ \\
\hline 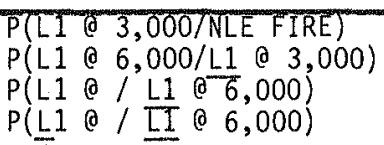 & $\begin{array}{r}.9189919 \\
8.1 \times 10-6 \\
0.0 \\
.0810\end{array}$ \\
\hline & SUM= \\
\hline
\end{tabular}
Room 1, the vast majority of that probability of being limited would fall within the $3000 \mathrm{sq}$. ft. floor area. Therefore, the succeeding sector probabilities would be much smaller; a pproaching zero at the overali room area.

Figure 3 SECTOR PROBABILITIES

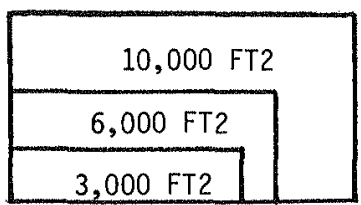

SECTORS

\section{DECISION MAKING AND THE ATTRACTIVE ALTERNATIVE}

With the knowledge of the probabilities in Figure 3 , a decision can now be made on which fire protection engineering alternative to implement. It can be shown that in every decision, there are three types of attitudes toward risk:

$$
\begin{aligned}
& \text { 1) risk aversion } \\
& \text { 2) risk neutrality } \\
& \text { 3) risk seeking }
\end{aligned}
$$

When a decision involves uncertainty, a rational decision maker is not risk neutral. Adecision maker in a business environment, again, if rational; is not risk seeking and therefore is risk averse. Lending upon corporate attitude toward risk, market conditions, and the size of the company, different degrees of risk aversion at different times will be evident. The risk attitude of the decision maker can be described by a risk constant, $r$, which enables a normalizing of all risk management alternatives.

on ce all alternatives have been normalized, the most attractive alternative is identified based on maximizing gain or minimizing loss. The 
risk constant reflects the risk attitude of the decision maker. As the risk constant changes, the degree of the risk attitude exponentially changes. If $r>0$, the decision maker is risk averse; if $r=0$, risk neutral; and if $r<0$, risk seeking.

Weighing is done using the following exponential utility relationship to determine the certainty equivalent (CE) of an alternative:

$$
C E=-1 / r \ln [E V(\exp -r z)]
$$

where $\mathrm{EV}$ is the individual expected values of each alternative. Alternatives are normalized by determining a CE. Expected value (EV) is simply an outcome of all possible outcomes, losses or gains, weighed by their probabilities of occurrence. The risk attitude of the decision maker is not considered in the EV calculation. Therefore the CE of an alternative, which considers risk attitude, is desired.

Decision making based on expected value criteria shows the risk neutral attitude. But, risk management decisions must consider associated uncertainty. Given the risk constant of the decision maker, the basis on which a decision is made can now be rationally changed. Selection of the most attractive risk management alternative can now be made based on the incorporated risk aversion of the decision maker. The CE calculation incorporates the decision maker's attitude toward relative amounts of money, or in other words, risk. The new decision criteria is now that of maximizing gain/minimizing loss.

\section{EVALUATING THE ALTERNATIVES}

The decision tree for the STATUS QUO (field evaluation) situation for the building of Figure 2 consists of two possibilities. Given established burning (EB*) in the following example, either a NLE fire can occur or a MFL fire can occur. The decision tree reflecting value exposed in the STATUS QUO situation under a NLE fire is provided by Figure 4. The MFL analysis branching off this tree is shown in Figure 5 . Note that this example only considers NLE and MFL fires.

The A-curve assessment of a MFL fire, P(MFL), is obtained from a decomposed probability technique by evaluating those events which can impair sprinkler system operation. In a similar manner, the complete L-curve is obtained, and the probability $P(N L E)$ is simply

$$
1-P(M F L)=P(N L E)
$$

as these events are mutually exclusive. Therefore, the fire risk presented by the STATUS QUO situation is the accumulation of NLE and MFL fire risks. The respective values at the end of each branch are negative because they represent expenditures either as a fire loss or as the cost to carry out a fire protection engineering recommendation, as the case may be.

(*) $E B$ is the size fire which threatens the building; usually taken as $250 \mathrm{~mm}$. $E B$ can change depending on the occupancy and construction of the building being addressed. 
Resulting from the original engineering survey which generated Figures 4 and 5 , three engineering solutions have been of fered to reduce the loss potential of this risk. Each alternative can be implemented either alone or together, at some respective cost. How does the fire risk manager evaluate the cost effectiveness of each alternative and thus quantify the resulting reduction in risk?

A cost effectiveness analysis for an alternative and the STATUS QOU situation would be done exactly the same. The post-survey engineering analys is of the property results in engineering recommendations. These must be evaluated to determine which offers the most increased protection, decreased insurance costs, favorable affect on risk acceptability, etc; for the cost of implementing the recommendation. These recomendations result in the following modified input data for the engineering method:

\section{ALTERNATIVE 1}

ROOM 1: $\quad I=0.6$

The fuel loading (stored combustibles) of Room 1 is either eliminated or rearranged such that flame spread is hampered or eliminated. COST TO IMPLEMENT: $\$ 0$

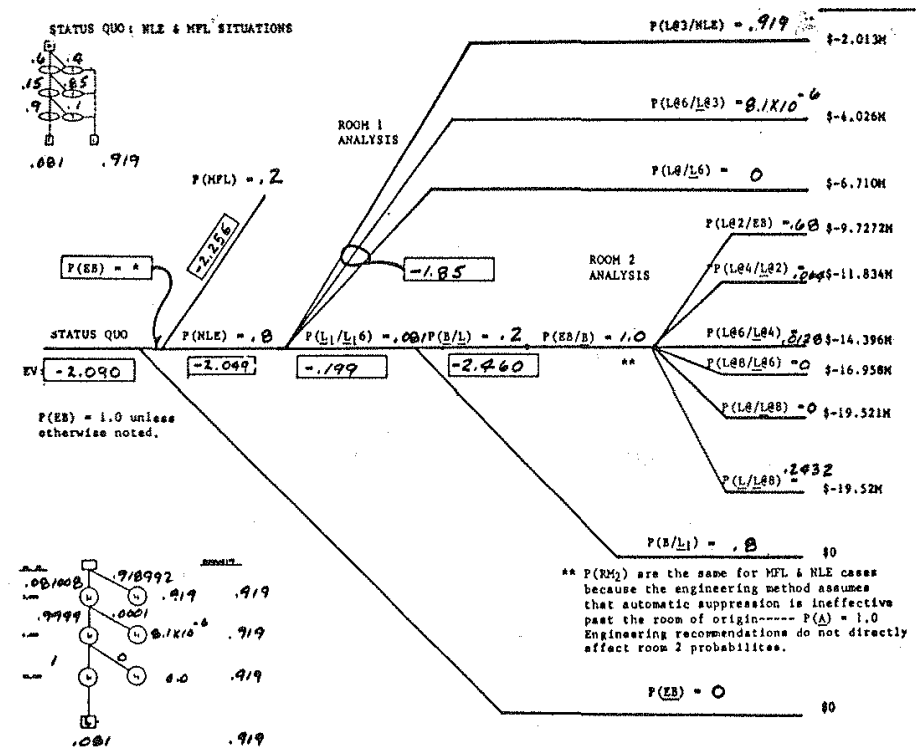

Figure 4 NLE Analys is For STATUS QUO

\section{ALTERNATIVE 2}

ROOM 1: $\quad I=0.6 \& M=0.2$

In addition to the above, improvement in detection is made by the installation of smoke and $f 1$ ame detectors. COST TO IMPLEMENT: $\$ 50,000$. 


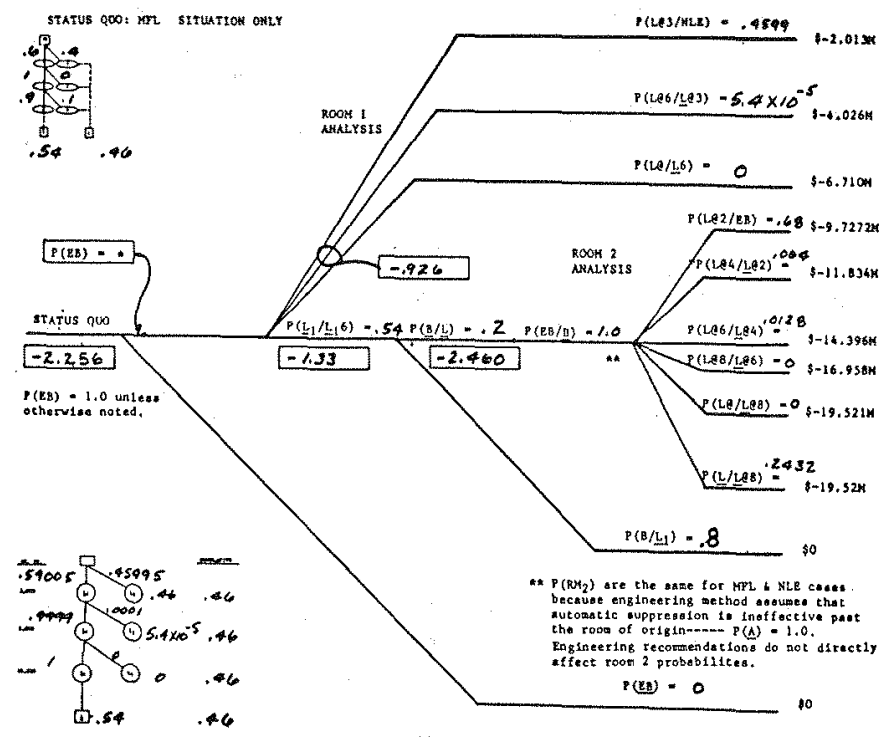

Figure 5 MFL Analysis For STATUS QUO

\section{ALTERNATIVE 3}

$$
P(M F L)=0.05 ; \text { ROOM } 1: I=0.6 . M=0.2
$$

Sprinkler valve maintenance program is implemented and complete electronic valve monitoring is acheived by installation of a new system. COST TO IMPLEMENT: $\$ 25,000$.

These modifications result in a change in network probability calculations of the engineering method. The resulting new probabilites are inserted into decision tress of the respective alternatives. The trees are rolled back to obtain an expected valve at the front of each tree for respective MNL \& MML cases of the above three engineering alternatives (4). Note, a modified NLE is a MNL; a modified MFL, is a MML.

An expected value ranking of these three engineering options and the STATUS QUO is:

$$
\text { EV RANK ING }
$$
1) ALTERNATIVE 1: $\$-2.0616 \mathrm{M}$
2) ALTERNATIVE 3: $\$-2.0639 \mathrm{M}$
3) STATUA QUO : $\$-2.0904 M$
4) ALTERNATIVE 2: \$-2.1046M

Table 6 EXPECTED VALUE RANKING

If the decision maker is neutral towards $r i s k, r=0$, the most attractive option of the four alternatives is Alternative 1 because it shows the least loss. However, as previously discussed, if uncertainty is invovled a rational 
decision maker is not risk neutral. The EV ranking of Table 6 does not consider the aversion to the uncertainty associated with loss potentials.

Risk aversion of the decision maker (corporation/risk manager) must now be included in the evaluation of the four alternatives. Given the range of loss potentials for the building of Figure 2 are,

and

\$-19.52M: total amount subject to loss

$\$-50,000$ : greatest expenditure of any one alternative,

two different risk constants, $r$, are determined:

$$
\begin{aligned}
& r(1)=1.25 \times 10-7 \\
& r(2)=1.00 \times 10-5
\end{aligned}
$$

Substituting the EV values for the four alternatives into equation 1 , based on respective risk constants, the four risk management options under consideration now fall into new rankings of attractiveness:

\section{$r=1.25 \times 10-7:$ LESS CONSERVATIVE}

1) ALTERNATIVE $1:$ CE $=\$-2.0617 \mathrm{M}$

2) ALTERNATIVE 3: CE $=\$-2.0697 \mathrm{M}$

3) STATUS QUO : $\mathrm{CE}=\$-2.0908 \mathrm{M}$

4) ALTERNATIVE 2: CE $=\$-2.1057 \mathrm{M}$ $r=1.00 \times 10-5:$ MORE CONSERVATIVE
1) ALTERNATIVE 3: CE $=\$-2.069 \mathrm{M}$
2) ALTERNATIVE 1: $C E=\$-2.075 \mathrm{M}$
3) ALTERNATIVE 2: CE $=\$-2.116 \mathrm{M}$
4) STATUS QUO: $\mathrm{CE}=\$-2.136 \mathrm{M}$

Additional $r$ values could yield yet different rankings of these alternatives. If the risk constant accurately describes the risk attitude of the decision maker, then the best engineering alternative is identified.

SUMMARY

An engineering method exists today for numerically evaluating a relative level of risk in any building. The flame movement part of the method involves the determination of the probability of success in terminating a fire within each space of a building. The effectiveness of each barrier surrounding the space, whether it be a floor, ceiling, wall, or an empty void; is also evaluated. A computer model exists which will describe any or all possible fire propagation paths from any specified room of origin and quantify the threat to any space along the path. The model then will calculate coordinates of the L-curve in time and space for each fire propagation path.

Once the probabilistic description of how the building in the example would react in a fire was determined, a decision analysis of possible fire protection alternatives was performed. This decision analysis evaluated the effectiveness of each fire protection alternative, and its cost to implement, against the status quo by incorporating the decision maker's (corporation/individual) willingness to accept risk associated with minimum and maximum loss potentiais.

The case study has been offered to demonstrate the incorporation of the engineering method into a decision analysis of possible alternatives based on 
the risk management attitude of the decision maker. This case study offers how the three risk management options of loss transfer, loss absorption or loss reduction can be considered rationally and analytically.

\section{REFERENCES}

(1) Fitzgerald, Robert. Risk Analysis Using the Engineering Method for Building Analysis. Worcester Polytechnic Institute, 1985.

(2) A computer program currently being developed by Professor Robert Fitzgerald, Worcester Polytechnic Institute; Worcester, Massachusetts.

(3) \& (4) Van Anne, Craig. Managing Risk Through Decision Analysis to Evaluate Firesafety Hazards and Extent of Probable Loss by Use of an Engineering Method. Worcester Polytechnic Institute, 1985. 